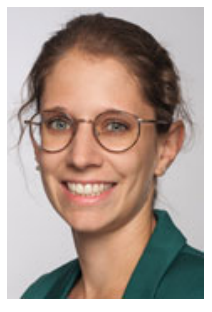

Iria Grundling

(geb. Bernhardsgrütter)

2010-2016 Biologiestudium an der ETH Zürich, Schweiz. 20162020 Promotion am Max-PlanckInstitut für Terrestrische Mikrobiologie in Marburg bei Prof. Dr. Tobias Erb. Seit 2020 Senior

Protein Engineer bei CarboCode Germany $\mathrm{GmbH}$, Konstanz.

DOI: $10.1007 / \mathrm{s} 12268-021-1578-4$

(C) Die Autorin 2021

- In Mikroorganismen entstanden im Laufe der Evolution verschiedene Enzyme und Stoffwechselwege zur $\mathrm{CO}_{2}$-Fixierung, die uns als Inspiration zur nachhaltigen Umwandlung von $\mathrm{CO}_{2}$ dienen können. Ein autotropher Stoffwechselweg ist der 3-HydoxypropionatBizyklus, mit seinem Schlüsselenzym Propionyl-CoA-Synthase (PCS) [1]. PCS ist ein Fusionsenzym aus drei Domänen, das die Umwandlung von 3-Hydroxypropionat in Propionyl-CoA katalysiert (Abb. 1A).

Proteinkristallographie der PCS aus Erythrobacter sp. NAP1 offenbarte im Innern des Enzyms eine Kammer, die von den drei Domänen (Ligase, Dehydratase und Reduktase) umschlossen wird und alle drei aktiven Zentren enthält (Abb. 1C, [2]). Wir konnten zeigen, dass die gesamte Reaktionskaskade innerhalb dieser Kammer abläuft, ohne dass Zwischenprodukte freigesetzt werden. Auf diese Weise wird verhindert, dass das toxische Zwischenprodukt Acrylyl-CoA im Cytoplasma akkumuliert. Mit einem Volumen von nur $33 \mathrm{~nm}^{3}$ ist die Proteinkammer der PCS die kleinste bisher beschriebene selbstorganisierte Multireaktionskammer und repräsentiert eine neue Strategie, um reaktive Zwischenprodukte zu kanalisieren.

Doch wie gelangen Substrate und Produkte aus der Reaktionskammer? Wir identifizierten die Ligase-Domäne als Gatekeeper. Die CoA-Ligase durchläuft während ihrer Reakti-
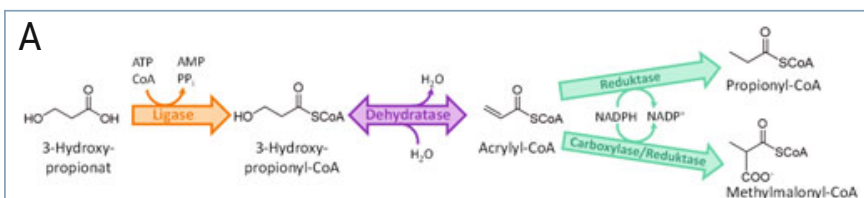

B

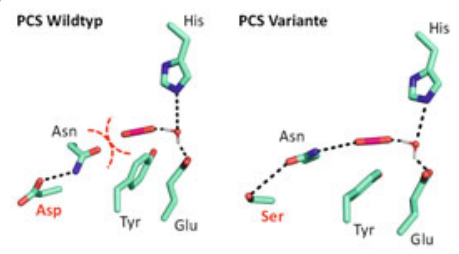

C

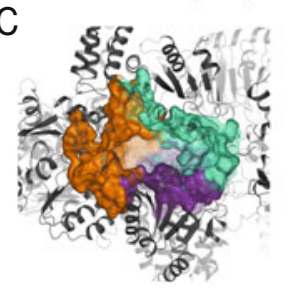

$\Delta$ Abb. 1: Trifunktionielle Propionyl-CoA-Synthase. A, Die drei Domänen (Ligase, Dehydratase und Reduktase) katalysieren die Reaktionskaskade von 3-Hydroxypropionat zu Propionyl-CoA bzw. Methylmalonyl-CoA. B, Ein Aspartat blockiert das Asparagin des aktiven Zentrums in einer Fehlorientiertung. C, Blick in die Reaktionskammer nach [2]. Ligase: orange; Dehydratase: violett; Reduktase: grün.

\title{
VAAM-Promotionspreis 2021
}

\section{Propionyl-CoA-Synthase: ein Nanoreaktor mit Megapotenzial}

IRIA GRUNDLING

MAX-PLANCK-INSTITUT FÜR TERRESTRISCHE MIKROBIOLOGIE, MARBURG

on eine Konformationsänderung, welche die Öffnung und Schließung der Reaktionskammer bewirkt. Diese hoch koordinierte Dynamik stellt sicher, dass das reaktive Zwischenprodukt nur in der geschlossenen Reaktionskammer entsteht, und macht die PCS zu einem wahrlich raffinierten Nanoreaktor.

Die Reduktase-Domäne der PCS weist hohe Ähnlichkeit mit Enzymen aus der Klasse der Enoyl-CoA-Carboxylasen/Reduktasen (ECR) auf. ECRs sind höchst effizient in der $\mathrm{CO}_{2}-$ Fixierung. Der Grund dafür liegt in einer ausgereiften $\mathrm{CO}_{2}$-Bindungstasche, worin das $\mathrm{CO}_{2}$-Molekül optimal für die Reaktion positioniert wird [3]. Zudem ist das aktive Zentrum vom umgebenden Wasser abgeschirmt, das andernfalls das $\mathrm{CO}_{2}$ in seiner Rolle als Elektrophil verdrängen kann. Überraschenderweise entdeckten wir die konservierten Aminosäuren dieser Bindungstasche auch in der Reduktase-Domäne der PCS, was uns eine bisher unbekannte Aktivität der PCS als Carboxylase vermuten ließ.

In Gegenwart von $\mathrm{CO}_{2}$ konnten wir tatsächlich Methylmalonyl-CoA als carboxyliertes Endprodukt der PCS nachweisen, allerdings zu einem sehr geringen Anteil von ungefähr drei Prozent. In den restlichen 97 Prozent katalysiert PCS die Reduktion zu PropionylCoA (Abb. 1A). Ein genauer Vergleich der $\mathrm{CO}_{2}$-Bindungstasche in PCS und ECRs zeigt Unterschiede in der zweiten Schale um das aktive Zentrum. Molekular-dynamische Simulationen bestätigten, dass diese Unterschiede zur Fehlorientierung eines Asparagins im aktiven Zentrum führen, was destabilisierend auf die $\mathrm{CO}_{2}{ }^{-}$ Bindung wirkt (Abb. 1B).

Durch gezielte Mutation konnten wir die korrekte Ausrichtung des Asparagins wiederherstellen, wodurch sich die Effizienz der $\mathrm{CO}_{2}$-Fixierung um das Siebenfache steigerte. Eine weitere Mutation außerhalb des aktiven Zentrums minimiert den Eintritt von Wasser in die $\mathrm{CO}_{2}$-Bindungstasche und somit die Reduktionsaktivität. Die Kombination beider Mutationen resultierte in einer PCS-Variante, die in 95 Prozent aller Reaktionen $\mathrm{CO}_{2}$ fixiert [4]. Damit gelang es uns, einen echten $\mathrm{CO}_{2}$-fixierenden Nanoreaktor zu erzeugen und den Kreis vom fundamentalen Verständnis der natürlichen $\mathrm{CO}_{2}$ Katalyse zur Konstruktion neuer $\mathrm{CO}_{2}$-Biokatalysatoren erfolgreich zu schließen.

\section{Danksagung}

Ein herzliches Dankeschön geht an Tobias Erb und die ganze Arbeitsgruppe. Vielen Dank auch an die verschiedenen Kollaborationspartner und an SFB 987 und IMPRS für die finanzielle Unterstützung.

\section{Literatur}

[1] Zarzycki J, Fuchs G (2011) Coassimilation of organic substrates via the autotrophic 3-hydroxypropionate bi-cycle in Chloroflexus aurantiacus. Appl Environ Microbiol 77: 6181-6188

[2] Bernhardsgrütter I, Vögeli B, Wagner T et al. (2018) The multicatalytic compartment of propionyl-CoA synthase sequesters a toxic metabolite. Nat Chem Biol 14: 1127 [3] Stoffel GMM, Saez DA, DeMirci H et al. (2019) Four amino acids define the $\mathrm{CO}_{2}$ binding pocket of enoyl-CoA carboxylases/reductases. PNAS 116: 13964-13969

[4] Bernhardsgrütter I, Schell K, Peter DM et al. (2019) Awakening the sleeping carboxylase function of enzymes: engineering the natural $\mathrm{CO}_{2}$-binding potential of reductases. JACS 141: 9778-9782

Funding note: Open Access funding enabled and organized by Projekt DEAL. Open Access: Dieser Artikel wird unter der Creative Commons Namensnennu 4.0 International Lizenz veróffentlicht, welche die Nutzung, Vervielfaltigung erlaubt, sofern Sie den/die ursprünglichen Autor(en) und die Quelle erlaubt, sofern Sie den/die ursprünglichen Autor(en) und die Quelle
ordnungsgemäß nennen, einen Link zur Creative Commons Lizenz beifügen und ordnungsgemäß nennen, einen Link zur Creative Commons Lizenz beifu
angeben, ob Änderungen vorgenommen wurden. Die in diesem Artikel angeben, ob Änderungen vorgenommen wurden. Die in diesem Artikel genannten Creative Commons Lizenz, sofern sich aus der Abbildungslegend nichts anderes ergibt. Sofern das betreffende Material nicht unter der genannten Creative Commons Lizenz steht und die betreffende Handlung nich nach gesetzlichen Vorschriften erlaubt ist, ist für die oben aufgeführt Weiterverwendungen des Materials die Einwilligung des jeweiligen Rechteinhabers einzuholen. Weitere Details zur Lizenz entnehmen Sie bitte de

Korrespondenzadresse:

Dr. Iria Grundling

Max-Planck-Institut für Terrestrische Mikrobiologie Karl-von-Frisch-Straße 10

D-35043 Marburg

Iria.grundling@mpi-marburg.mpg.de

CarboCode Germany $\mathrm{GmbH}$

Byk-Gulden-Straße 2

D-78467 Konstanz

iria.grundling@carbocode.com 